\title{
Interprofessional Research Team Approach Is the Key to Traumatic Brain Injury Intervention
}

\author{
Ranabir Pal ${ }^{1, \odot}$ \\ ${ }^{1}$ Department of Community Medicine, MGM Medical College and \\ Hospital, Kishanganj, Bihar, India
}

J Neurosci Rural Pract 2020;11:3-4

The spectrum of traumatic brain injury (TBI) has been considered as global public health menace with important impacts on cognition, emotional, and psychosocial health, particularly in post-mild TBI. The "Position Paper: Recommendations of the Colombian Consensus Committee for the Management of Traumatic Brain Injury in Prehospital, Emergency Department, Surgery, and Intensive Care (Beyond One Option for Treatment of Traumatic Brain Injury, A Stratified Protocol [BOOTStraP])" has depicted transparent roadmap. ${ }^{1}$ The burden of TBI is increasing in the low- and middle-income countries (LMICs) parallel with the explosion of science and technological inventions and discoveries, as work related TBI is more than half of reported cases. Though one in five TBI is reported as major injuries, more frequent victims are from younger age groups inclusive of prehospital and postinjury hitches. There is an array of outcomes of morbidity, mortality, and disability with all forms of damage in and around head from known and unknown hazards leading to the overall brunt more than direct and immediate impacts. There are indeterminate incremental-associated socioeconomic burdens with stress on already overburdened general health care services, particularly in the LMICs. There is abundant literature on different clinical aspects of the spectrum of TBI. Yet, we have noted paucity of research studies on holistic approach on the management of TBI. Also in many places, even if TBI care facilities are available adequately, there is reluctance in its use by the primary health care providers based on ignorance on the transparent limiting steps of dos and don'ts. As such, there is more need of creating awareness among population in this regard in a concerted manner. This position paper from Colombian Consensus Committee is definitely going to help researchers of LMICs to formulate clinical practice guidelines for their respective countries. ${ }^{2-4}$

Understanding and treating of head injuries embrace internalization that it is a spectrum of sudden, unexpected, unintended, and violent deleterious consequences affecting head region. The basic pathophysiology and current evidences indicate that TBI is absolutely preventable and manageable emergency persisting as the hidden epidemic

of modern society. The discrepancy in the precise description and classification of TBI, along with inconsistency in data collection in absence of trauma registry in LMICs, has made the epidemiology of head injury difficult to internalize. Research on TBI had been performed globally for decades noted that mild TBI or concussion cases are usually managed by primary care physicians, and severe, as well as fatal, cases are only recorded by the stakeholders. So, to understand the growing need, we need to adopt innovative techniques for TBI managements embedded with the qualitative research directions in search of well-designed interventions. Thus, scientifically sound studies, using multipronged approaches with achievable research questions to test hypotheses and to produce generalizable futuristic observations, can develop well-designed interventions. The finding of TBI research indicated that the perceptions toward interventions could be influenced to a large extent by knowledge significantly related with the occupation and education among the general population. The proposition that early recognition of the extent and pattern of TBI can improve scopes for holistic solutions those are easier said than done. It is important that health care providers including family practitioners should be involved and trained in the diagnosis and treatment of TBI from undergraduate health care capacity building. Supporting TBI patients and their caregivers, empathetically, to ensure that they understand the limitation of these treatments, remains an important component of TBI management.

TBI is taking tolls despite sincere implementation of effective protected strategies and establishment of state-of-art centers of neurosciences manned by experts in the fields. Real-life internalization on specific TBI risk is revitalized at every moment with the passing of days in ice-ball phenomenon by encouraging competency-based training with stress on skill domain in the nonthreatening environment and rewarding positive performance. Successful TBI control envisages on futuristic vision and proactivities ranging from recognition of hazards to the empathetic implementation of injury surveillance that includes injury registry, mitigation, plan, and control programs followed by holistic evaluation.

C2020 Association for Helping
Neurosurgical Sick People
License terms

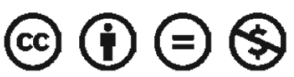

Address for correspondence Ranabir Pal, MBBS (Hons.), MD, PhD, DCH, MBA, MNAMS, MNASc, Department of Community Medicine, MGM Medical College and Hospital, Kishanganj, Bihar 855107, India

(e-mail: ranabirmon@yahoo.co.in).
DOI https://doi.org/ 10.1055/s-0040-1701540 ISSN 0976-3147. 
An understanding of the interprofessional (IP) research team involving experts of all walks of life, viz., physician, injury care personnel, physical medicine expert, rehabilitation therapist, social scientists, psychologists, educationists, and technical and technological experts among others is essential component of successful TBI control. An ideal clinical practice guideline with combination of stepwise medical and surgical managements at the nonneurosurgical centers can save millions of lives. We need more qualitative researches in the continuum under leadership of interprofessional team involving persons from all aspects of life as the call of the day. Truthfully establishing linkages of the concerned persons of multiple domain outside the neurosciences community can solve this mammoth task with multipronged approach. ${ }^{5,6}$

\section{Funding}

None.

\section{Conflict of Interest}

None declared.

\section{References}

1 Rubiano AM, Vera DS, Montenegro JH, et al. Recommendations of the Colombian Consensus committee for the management of traumatic brain injury in prehospital, emergency department, surgery, and intensive care (beyond one option for treatment of traumatic brain injury, a stratified protocol [BOOTStraP]). J Neurosci Rural Pract 2020;11(1): 7-22

2 American College of Surgeons Committee on Trauma. ACS TQIP Best Practice guidelines. Available at: https://www.facs. org/quality-programs/trauma/tqp/center-programs/tqip/ best-practice. Accessed January 15, 2020

3 Cole WR, Bailie JM. Neurocognitive and psychiatric symptoms following mild traumatic brain injury. In: Laskowitz D, Grant G, eds. Translational Research in Traumatic Brain Injury. Boca Raton, FL: CRC Press/Taylor and Francis Group; 2016

4 Dawodu ST. Traumatic brain injury (TBI)-definition, epidemiology, pathophysiology. Available at: https://emedicine.medscape.com/article/326510-overview\#a2. Accessed October 20, 2019

5 Pal R, Agarwal A, Galwankar S, et al. The 2014 Academic College of emergency experts in India's INDO-US joint working group (JWG) white paper on "developing trauma sciences and injury care in India" Int J Crit Illn Inj Sci 2014;4(2):114-130

6 Nanclares BVC, Padilla-Zambrano HS, El-Menyar A, et al. WACEM consensus paper on deep venous thrombosis after traumatic spinal cord injury. J Emerg Trauma Shock 2019;12(2):150-154 